(C) by R. Oldenbourg Verlag, München 1997

\title{
Crystal structure of indium magnesium tribromide, $\operatorname{InMgBr}_{3}$
}

\author{
M. Scholten and R. Dronskowski \\ Max-Planck-Institut für Festkörperforschung. Heisenbergstraße I, D-70569 Stuttgart. Germany
}

Received June 24. 1996. transferred to 2nd update of database ICSD in 1997, CSD-No. 402533

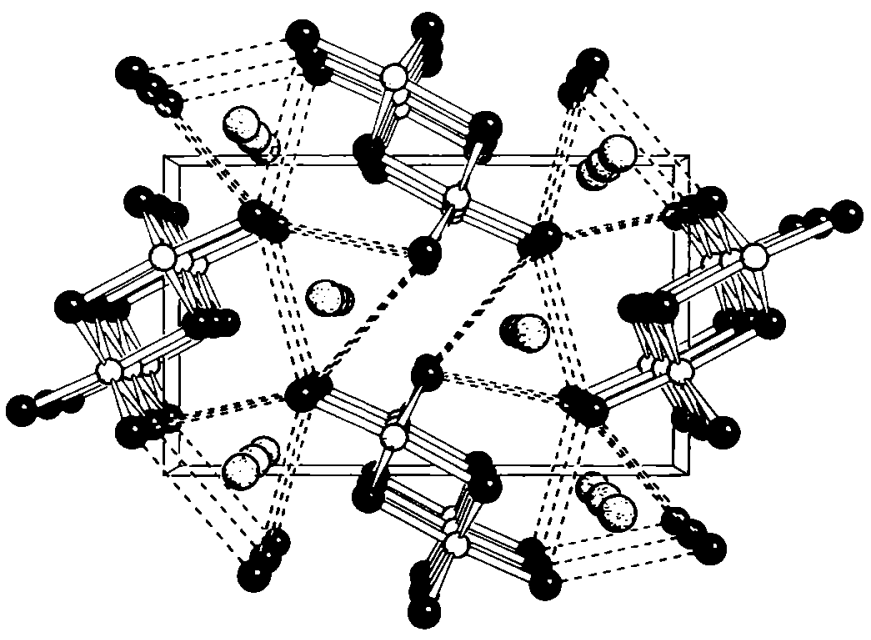

Fig. 1. [010] view with In as large shaded circles, $\mathrm{Mg}$ as small open circles, and $\mathrm{Br}$ as medium-sized black circles.

Source of material: Synthesis from molten $\mathrm{InBr}_{3}$ and elemental magnesium at $723 \mathrm{~K}$ for five days in evacuated glass ampoules, followed by slow cooling ( $2 \mathrm{~K} /$ hour).

InMgBr 3 crystallizes in the $\mathrm{NH}_{4} \mathrm{CdCl}_{3}$ structure type (see ref. 1), as do other ternary transition metal bromides containing univalent indium (see refs. 2,3 ). While magnesium is octahedrally coordinated by bromine $(262 \mathrm{pm}-273 \mathrm{pm})$, univalent indium resides in a strongly distorted trigonal prism of bromine, tricapped by additional bromine ions (eight bonds between $330 \mathrm{pm}$ and $353 \mathrm{pm}$, one distance of $420 \mathrm{pm}$ ).

Br3InMg, orthorhombic, Pnma (No. 62), $a=9.4395(1) \AA$, $b=3.9538(1) \AA, c=15.3744$ (2) $\AA, V=573.8 \AA^{3}, Z=4$, $\rho_{\mathrm{m}}=4.390 \mathrm{~g} \cdot \mathrm{cm}^{-3}, R_{p}=0.028, R_{w p}=0.036, R_{B r a g g}=0.02$, $R_{\text {exp. }}=0.028$.
Table 1. Parameters used for the $\mathrm{X}$-ray data collection

\begin{tabular}{|c|c|}
\hline Powder: & $\begin{array}{l}0.1 \mathrm{~mm} \text { capillary of powdery, transparent } \\
\text { material (ground needles) }\end{array}$ \\
\hline Wavelength: & Cu $K_{\mathrm{al}}$ radiation $(1.54056 \AA)$ \\
\hline & $249.50 \mathrm{~cm}^{-1}$ \\
\hline $\begin{array}{l}\text { Diffractometer: } \\
\text { Sample geometry }\end{array}$ & Stoe STADI powder diffractometer \\
\hline Scan mode: & $2 \theta$ \\
\hline$T_{\text {measurement: }}$ & $293 \mathrm{~K}$ \\
\hline $2 \theta_{\max }:$ & $95^{\circ}$ \\
\hline $\mathrm{N}(\text { points })_{\text {meas }}$ : & 8500 \\
\hline $\mathrm{N}(\text { param })_{\text {refined: }}$ & 26 \\
\hline Profile function: & Pseudo Voigt \\
\hline Background correction: & manual + Fourier filtering \\
\hline Program: & Rietveld DBW9006 \\
\hline
\end{tabular}

Table 2. Final atomic coordinates and displacement parameters (in $\AA^{2}$ )

\begin{tabular}{llllll}
\hline Atom & Site & $x$ & $y$ & $z$ & $B_{\text {iso }}$ \\
\hline In & $4 c$ & $0.4502(1)$ & $1 / 4$ & $0.6764(1)$ & $3.01(4)$ \\
$\operatorname{Mg}$ & $4 c$ & $0.1619(5)$ & $1 / 4$ & $0.4457(3)$ & $1.3(1)$ \\
$\operatorname{Br}(1)$ & $4 c$ & $0.6738(1)$ & $1 / 4$ & $0.4979(1)$ & $0.90(4)$ \\
$\operatorname{Br}(2)$ & $4 c$ & $0.2755(1)$ & $1 / 4$ & $0.2905(1)$ & $1.47(5)$ \\
$\operatorname{Br}(3)$ & $4 c$ & $0.0228(2)$ & $1 / 4$ & $0.6008(1)$ & $0.80(4)$ \\
\hline
\end{tabular}

\section{References}

1. Brasseur, H.; Pauling, L.: The Crystal Structure of Ammonium Cadmium Chloride, $\mathrm{NH}_{4} \mathrm{CdCl}_{3}$.. J. Am. Chem. Soc. 60 (1938) 2886-2890.

2. Dronskowski, R.: InFeBr3 and InMnBr3: Synthesis, Crystal Structure, Magnetic Properties and Electronic Structure. Inorg. Chem. 33 (1994) 5927-5933.

3. Dronskowski, R.: Synthesis, Crystal Structure, and Electronic Structure of InCdBr3. J. Solid State Chem. 116 (1995) 45-52.

4. Young, R. A.: Program for Rietveld Analysis of X-Ray and Neutron Powder Diffraction Patterns; DBW9006; School of Physics, Georgia Institute of Technology, Atlanta, GA 30322. 